\title{
The effect of overtraining on reversal learning under conditions of no nonreinforcement'
}

SANFORD FIDELL AND DAVID BIRCH

UNIVERSITY OF MICHIGAN

Groups of rats trained with 60 or 198 trials on a one vs. three pellet $\mathrm{T}$-maze place discrimination task were subsequently taught the reverse of the task. During reversal the high incentive alternative contained nine pellets while the reward on the other side remained three pellets. The number of incorrect choices during reversal was directly related to amount of initial training. Significant increases and decreas$e s$ in running speeds to the high and low incentive alternatives, respectively, were observed during reversal training.

Early theorizing about the overlearning reversal effect (ORE) by Reid (1953), Pubols (1956), and Capaldi \& Stevenson (1957) was done in terms of acquired distinctiveness of cues, differential position habit strengths, and differences in patterns of reinforcement during original and reversal training. Subsequently the concept of frustration has come to play a prominent role in such theorizing (Birch, 1961; Amsel, 1962) and considerable evidence has been gathered pointing to the importance of frustration effects in ORE (Birch, Ison, \& Sperling, 1960; Clifford, 1964; Theios \& Brelsford, 1964; and Ison, 1962). Evidence demonstrating the relevance of one factor to a phenomenon does not, of course, rule out the possible relevance of other factors. With this in mind the present study was designed to provide a reversal learning setting in which frustration would not be expected to occur when reversal was instituted. An ORE obtained under these conditions would force consideration of factors other than those attributable to frustration for a complete account of the ORE.

The experiment employed a T-maze in which Ss were presented with three pellets of food in one arm and one pellet in the other during original learning. For "reversal" learning the three pellets in the one arm were continued and the number of pellets was increased from one to nine in the other arm, thereby eliminating or at least minimizing frustration. Finding an ORE under these conditions would be particularly striking since simple T-maze learning was involved and as Sperling (1965, p. 281) has pointed out, "...the ORE seems to be favored by the use of exteroceptive discriminative stimuli while training on a position response most frequently results in no ORE or a reverse ORE."

Method

Ss were 25 male hooded rats from the Michigan laboratories 60 days old at the beginning of experimentation.

Ss were run in a T-maze $4 \mathrm{in}$. wide and $4-1 / 2 \mathrm{in.} \mathrm{high}$ with 20-in. arms, a 13-in. stem and a 9-in. starting box, all covered with hinged hardware cloth. On each experimental day Ss received two initial free choice trials followed by four forced trials in which the door to one of the arms was lowered before $S$ was brought into the experimental room. Forced trials were administered according to four rotating patterns which balanced the number of trials to each side for each $\mathrm{S}$ each day. The minimum intertrial interval was $3 \mathrm{~min}$. Ss were run in a random order shortly after noon and $22 \mathrm{hr}$, after the last feeding, which consisted of free access to food for $1 \mathrm{hr}$., not less than $1 / 2 \mathrm{hr}$. after the last trial of the previous day. The feeding schedule was instituted two weeks before experimentation.

On each trial $S$ was placed head forward in the start chamber. At the count of 5 the guillotine door was raised quickly. If $\mathrm{S}$ entered an arm of the maze by one body length the gullotine door of the arm was lowered behind him. S was removed from the maze when he ate all the food pellets in the food cup. The elapsed time between the raising of the start box door and entering an arm of the maze was recorded to $.01 \mathrm{sec}$.

Eighteen Ss were run to a 10/10 correct choice criterion on the initial one vs. three pellet condition. As $S$ reached criterion he was randomly assigned to either the Overtraining (OT) or Storage (ST) group, and the next $S$ to reach criterion was put in the other group. Assignment of high and low incentive sides of the maze to Ss was also random.

OT Ss continued to run to an $18 / 20$ correct choice criterion, after which they recelved 168 additional trials on the original task. ST Ss were returned to their home cages for 28 days, handled dally before feeding, and otherwise treated as Ss in the OT group. At the end of the storage period ST Ss completed their 18/20 correct choice criterion on the initial discrimination. The storage procedure minimized age and experience differences at the time of reversal.

Reversal training for both groups started upon completion of initial training. During reversal the arm which had held one pellet contained nine pellets, but no other changes were made in trial procedure. A control (C) group of seven Ss was run to an $18 / 20$ correct choice criterion under these conditions with no prior training. OT and ST Ss were also run to $18 / 20$ correct choice criteria in reversal.

\section{Results and Discussion}

All groups reached criterion readily in original learning with only minor differences among groups in the number of trials required. Means were 75.36, 78.66, and 74.58 for the OT, ST, and C groups, respectively. The groups were also very similar in the mean number of correct choices made in reaching the reversal cri- 


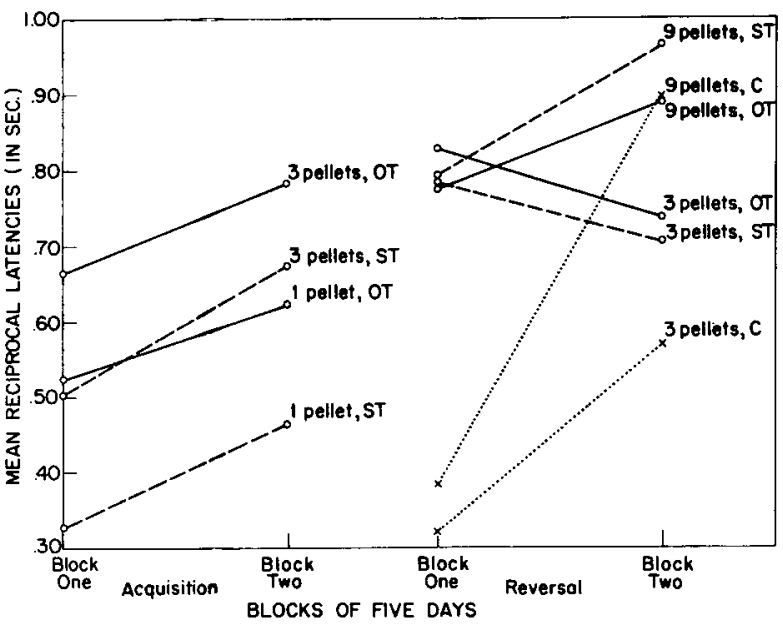

Fig. 1. Running speeds to the large and small incentive sides before and after reversal.

terion; $19.34,19.22$, and 19.42 for OT, ST, and C, respectively. There were major differences among groups, however, in the number of incorrect choices in reversal. The groups were ordered by amount of prereversal training, with the group given the most training making the most errors in reversal; the means were 12.67 (OT), 8.78 (ST), and 3.71 (C). Analysis of variance indicated the significance of the differences between groups ( $\mathrm{F}=$ $15.36, \mathrm{df}=2 / 22, \mathrm{p}<.01$ ), the differences between correct and incorrect choices $(F=207.22, d f=1 / 22, p<.001)$, and the Choice by Groups interaction $(F=10.55, \mathrm{df}=$ $2 / 22, \mathrm{p}<.01)$. An analysis of the incorrect choice data also showed significance for the differences among groups $(F=16.45$, df $=2 / 22, p<.01)$.

Two scores for each $\mathrm{S}$ on each day were picked from the forced choice trials, one each from the low and high incentive trials, for the analysis of the latency data. The scures were picked according to four patterns which counterbalanced order of occurrence of the low and high incentive trials. Means of the reciprocals of these scores (running speeds) for all three groups over fiveday blocks at the end of acquisition and the beginning of reversal training are plotted in Fig. 1.

In analyzing the data for OT and ST, the running speeds for $\mathrm{Ss}$ in reversal training were adjusted for running speed levels at the end of initial training. Analysis of variance comparing speed of running to the three and nine pellets arms on the second block of trials shows that learning had occurred by that point $(F=39.63, \mathrm{df}=$ $1 / 16, p<.001)$. There is no indication that the OT and ST groups learned the reversal task at different rates, as the triple interaction, Incentive by Blocks by Groups, is very small $(\mathrm{F}=0.08, \mathrm{df}=1 / 16)$. The Incentive by Blocks interaction is significant $(F=10.86, \mathrm{df}=1 / 16$, $\mathrm{p}<.01$ ) and results from the downward trend of speeds to the three pellet side in conjunction with the upward trend to the nine pellet side over blocks of trials. No other effects were signiflcant in this analysis.

The choice data are in agreement with Sperling's (1965) conclusion that overtraining on a position discrimination task does not foster an ORE; in fact, a reverse ORE was found in the current experiment. The latency data further corroborate Sperling's suggestion, since there was no evidence of differences in running speeds between the OT and ST groups over blocks of trials during reversal training.

\section{References}

Amsel, A. Frustrative nonreward in partial reinforcement and discrimination learning: Some recent history and a theoretical extension. Psychol. Rev., 1962, 69, 306-328.

Birch, D., Ison, J., \& Sperling, S. Reversal learning under single stimulus presentation. J. exp. Psychol., 1960, 60, 36-40.

Birch, D. A motivational interpretation of extinction. In M. R. Jones (Ed.), Nebraska Symposium on Motivation. Lincoln: University of Nebraska Press, 1961, Pp. 179-201.

Capaldi, E., \& Stevenson, H. Response reversal following different amounts of training. J. comp. physiol. Psychol., 1957, 50, 195198

Clifford, L. T. Extinction following continuous reward and latent extinction. J, exp. Psychol., 1964, 68, 456-465.

Ison, J. R. Experimental extinction as a function of number of reinforcements. J. exp. Psychol., 1962, 64, 314-317.

Pubols, B. The facilitation of visual and spatial discrimination by overlearning. J. comp. physiol. Psychol., 1956, 49, 243-248.

Reid, $\mathbf{L}$. The development of non-continuity behavior through continuity learning. J. exp. Psychol., 1953, 46, 107-112.

Sperling, S. Reversal learning and resistance to extinction: A review of the rat literature. Psychol. Bull., 1965, 63, 281-297.

Theios, J., \& Brelsford, J. Overlearning-extinction effect as an incentive phenomenon. $J$. exp. Psychol., 1964, 67, 463-467. Note

1. This research was supported by a grant, NSF GB-2910, to the second author. 